\title{
TIME-DEPENDENT SOURCES IDENTIFICATION FOR TRANSMISSION LINES PROBLEMS
}

\author{
J. Benoit ${ }^{1,2,4}$, C. Chauvière ${ }^{1,3}$, P. Bonnet ${ }^{1,2}$, S. Girard ${ }^{1,2}$ \\ ${ }^{1}$ Clermont Université, Université Blaise Pascal, Institut Pascal, BP 10448, F-63000 Clermont-Ferrand \\ Pierre.Bonnet@lasmea.univ-bpclermont.fr \\ ${ }^{2}$ CNRS, UMR 6602, IP, F-63171 Aubière, France \\ ${ }^{3}$ CNRS, UMR 6620, Laboratoire de Mathématiques, F-63171 Aubière, France \\ ${ }^{4}$ CEA, LIST, F-91191 Gif-sur-Yvette, France
}

\begin{abstract}
This paper is devoted to introduce an extension to the Linear Combination of Configuration Fields (LCCF). This new numerical method was synthesized to compute the time profile of an electromagnetic source radiating a specified electromagnetic field in all or part of the computational domain, for a specified duration. There is no theoretical limit for this duration; however the computational time grows significantly as this parameter is increased. Here, we extend this idea within the framework of transmission lines network. The main advantage of the proposed method over the time reversal method is the possibility to find a source, whatever the physically acceptable field specified, even in a lossy medium. The principle of the method is first validated numerically. Then we prospect the same ideas in a realdata experiment which shows that the method is ready for real-life investigations. In that case, it is the impulse response of the line under test that is used to construct the matrix for the computation of the unknown source. Then, this source is injected into the network to check that it gives the desired signal at a point of the network.
\end{abstract}

\section{INTRODUCTION}

Research on inverse problems is both a wide and active domain of research that constitutes a subject in itself. Applied to electromagnetism, and more specifically to electromagnetic compatibility (EMC), an inverse problem consists in getting back to a set of parameters of the physical system, from the observation of data (in general electric or magnetic field, currents...) produced by that system. In the vast majority of cases, the parameters to be determined are linked to physical characteristics of the system: dielectric permittivity, conductivity, geometry of the diffracting objects... Less often, the authors have dedicated their researches to electromagnetic sources.

However, the identification of radiating sources through near-field to far-field transformations [5,8], or for the determination of equivalent sources $[6,7]$, is a field of research that gives rise to numerous studies and applications. Although those techniques are inherently frequency-domain, the need for time-domain results has pushed researchers to design mixed methods [19,20]. Another possibility for modeling problems by equivalent dipole is to use simplified analytical solutions (see [18], for example). Very few articles report source identification (field source or voltage source) in time domain. When it is the case, most of them use time reversal (TR) with possible enhancements. In [14], for example, the basic reversed-TLM method is improved in a second step by cancelling the divergent wave that occurs in TR numerical simulations.

In this paper, we are not concerned with existence or uniqueness of the solution to this inverse problem. The approach described here is pragmatic: should the proposed numerical method lead to a solution, then our only concern is to ensure that the identified source satisfies the fixed objectives, whether it be numerically or experimentally.

To be more specific, in this article we are concerned with the resolution of the following problem: in a transmission line network, what voltage should we use from a specified emission point in order to get a specified voltage at another given point of the network, over a certain period of time? The proposed method applies to all types of transmission lines networks (coaxial cable, twisted pair, microstrip, etc...) as far as they have a linear behavior.

After having treated this problem in a first part of the paper, we turn our attention to fault detection in networks. Although the two problems do not seem to be linked, in fact they can be efficiently used together. Since we are able to construct a source that creates a specified voltage at any point of the network, an interesting source would be one that amplifies any small changes in that network. Then, a comparison of the signal at a point of the network between the healthy and the faulty network can highlight any changes between the two. Here the point of comparison is not necessary the injection point. Our approach is rather different from the widely used reflectometry which consists in injecting a signal into the wiring network and analyzing the reflections at the injection point in order to detect and locate a fault. Several improvement of this basic technique have been made : use signal processing techniques and clustering [17], use testing signals that are network dependent [16], use time reversal [21] or more interestingly, solve an inverse problem from the measurements of the reflectometry response [15]. The reflectometry method and its refinements are generally good at detecting and locating hard faults but soft faults are still a challenge [22]. The method we propose is able to detect both soft 
and hard faults; however it does not have the capability to locate them.

The paper is organized as follows: in the next section, we briefly recall the principle of the linear combination of the configuration field in the context of electromagnetic source identification. Then, in section III, the same ideas are adapted to transmission lines problems. Numerical experiments of section IV show that the computed voltage source presents good properties for fault detection in a network. This finding is then corroborated by results of an experimental set up in section V. Finally, we draw some conclusions in section VI.

\section{THE BASIC LCCF (LINEAR COMBINATION OF CONFIGURATION FIELD) METHOD}

In [2], an original numerical method was introduced to get a temporal electromagnetic source that gives a specified electromagnetic field all over the domain, some time after the source starts emitting. Authors showed that the source could be obtained as a solution to a linear system and the method was tested on one and twodimensional problems. When the target field is a spatiotemporal focusing, comparisons with the time reversal (TR) method $[10,11]$ showed the superiority of the LCCF in terms of focusing and signal to noise ratio [1]. Furthermore, the LCCF is more general than the TR since it does not require the time reversibility of wave equations. Therefore it can deal with waves in lossy media. Another advantage of the LCCF in its basic version over the TR is the possibility offered by this method to obtain any spatially specified field more accurately (as far as it is physically acceptable) but still at a given instant of time. Typical results are shown on figure 1, for a 1D example, obtained after a source has emitted for a duration $T=10^{-8} \mathrm{~s}$ (see [2] for more details).

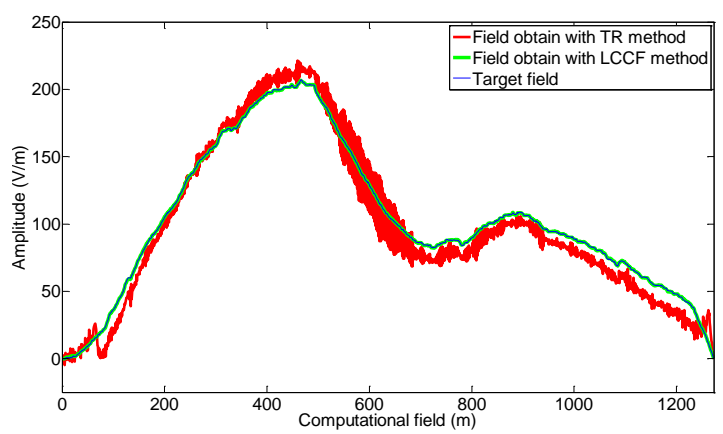

Fig.1: Comparison between the target electric field and the electric field obtained with the source computed with the LCCF method and the time reversal (TR) method.

\section{THE EXTENDED LCCF METHOD}

In this section, we develop an extension of the basic LCCF method [1,2] for which the source is built to obtain a specified field at a given point, but over a period of time instead of an instant of time.

\section{III.1. Objectives and context}

The approach described in this paper is quite general and could be applied to many EMC problems, as long as the governing equations are linear. Furthermore, it is independent of the solver used to discretize the equations. However, for the purpose of clarity, here we do not present the method for general linear differential equations but we introduce it within the framework of current/voltage propagation in lossy transmission lines. In that case, the integration of Maxwell's equations with usual simplifications leads to the so-called telegrapher equations [3] given by

$$
\begin{aligned}
& \frac{\partial v(z, t)}{\partial z}+\frac{L \partial i(z, t)}{\partial t}+R i(z, t)=0, \\
& \frac{\partial i(z, t)}{\partial z}+\frac{C \partial v(z, t)}{\partial t}+G v(z, t)=0,
\end{aligned}
$$

where $v=v(z, t)$ and $i=i(z, t)$ are the voltages and the currents, respectively, with $(z, t) \in \Omega \times[0, T] . \Omega$ is a general domain modeled within the framework of RLCG transmission lines (see [3] for details). R, L, C, G are the resistance, the inductance, the capacitance and the conductance, respectively, of the conductor per unit of length. The numerical resolution of (1) is performed using finite difference technique, with space discretization $\Delta z$ and time discretization $\Delta t$.

We note, $\mathbf{u}^{n}=\left[\mathbf{v}^{n}, \mathbf{i}^{n}\right]^{T}$ a vector containing the value of the voltage and the current at the points of discretization of the domain $\Omega$ at time $t_{n}=n \Delta t$. We also note $\mathbf{P}$, an operator that computes voltages and currents $\mathbf{u}^{n+1}$ at time $t_{n+1}$ from voltages and currents at time $t_{n}$, i.e. $\mathbf{u}^{n+1}=\mathbf{P} \mathbf{u}^{n}$. In practice, for a given set of equations (1), $\mathbf{P}$ is a matrix that only depends on the technique of discretization used (finite difference [9], finite volume [12], spectral method [13], ...). The objective is to obtain an expression of one (or several) voltage source leading to a specified voltage at another point of the network $\mathbf{z}_{a} \in \Omega$ during a certain period of time. For that, we place a voltage (or current) source at point $\mathbf{z}_{s} \in \Omega$ that starts emitting a signal at time $t_{p}=p \Delta t$. We also note $t_{p+q}=(p+q) \Delta t$ the instant of time from which we wish to force a specified voltage at point $\mathbf{z}_{a} \in \Omega$.

\section{III.2. The principe of the method}

As a first step, a source creates a wave that propagates and gets reflected in the network $\Omega$. That wave can be generated from any point of the network and take any form. The only purpose of this wave is to start the control process at point $\mathbf{z}_{a} \in \Omega$, with some non-zero values for the voltage and the current in the network. 
As target voltage, we wish to obtain $v\left(\mathbf{z}_{a}, t\right)=0$ for $t \in\left[t_{p+q}, t_{p+f}\right]$. However, any other physically acceptable profile could equally be set.

Let $b_{k}=v\left(\mathbf{z}_{a}, t_{k}\right)$, with $k \in\{p+q, \ldots, p+f\}$, denote the value of the voltage $v$ at point $\mathbf{z}_{a}$ and at time $t_{k}=k \Delta t$ produced by the initial source.

We assume that the voltage source to be computed has the following form :

$$
S_{v}(\mathbf{z}, t)=\sum_{k=p}^{p+f} s_{k} \mathbf{1}_{\left[t_{k}, t_{k+1}\right.}(t) \delta\left(\mathbf{z}-\mathbf{z}_{s}\right)
$$

where $\delta$ is the Dirac delta function and $\mathbf{1}_{\left[t_{k}, t_{k+1}[\right.}(t)$ is the indicatrix function defined by

$$
\mathbf{1}_{t_{t_{k}, t_{k+1}}[}(t)=\left\{\begin{array}{l}
1 \text { if } t \in\left[t_{k}, t_{k+1}[\right. \\
0 \text { otherwise }
\end{array}\right.
$$

Then, the unknowns of the problem are the coefficients $s_{k}$ of equation (2) above. Such an expression for the voltage source ensures that it is located at $\mathbf{z}=\mathbf{z}_{s}$.

We assume that the domain $\Omega$ is discretized into a set of $n_{v}$ points for the voltage and $n_{k}$ points for the current. For some numerical methods, $n_{v}=n_{k}$ but when a staggered grid is used, such as for the Finite Difference Time Domain (FDTD) method, $n_{v} \neq n_{k}$. We also assume that the location of the voltage source to be determined corresponds to the index $s$ of the vector $\mathbf{u}$ that contains the discrete values of the voltage and the current in the network. Denoting by $\mathbf{u}_{s}=(0 \ldots 010 \ldots 0)^{T}$ a vector from $R^{n_{v}+n_{k}}$ such that $\mathbf{u}_{s}(l)=\delta_{l s}$, the discrete equivalent of equation (2) at time $t_{k}$ can be written under the form of a vector as follows:

$$
\left(\mathbf{S}^{k}\right)^{T}=\left(\mathbf{S}_{v}^{k}, \mathbf{S}_{i}^{k}\right)^{T}=s_{k} \Delta t \mathbf{u}_{s}
$$

with $k \in\{p, \ldots, p+f\}$.

In order to justify the expression above, we recall that in the absence of sources, we advance from the field $\mathbf{u}^{\mathrm{n}}$ to the field $\mathbf{u}^{\mathrm{n}+1}$ through the matrix $\mathbf{P}$ according to $\mathbf{u}^{n+1}=\mathbf{P} \mathbf{u}^{n}$. In the presence of a source at time $\mathrm{t}_{\mathrm{n}+1}$, this expression has to be modified according to

$$
\mathbf{u}^{n+1}=\mathbf{P} \mathbf{u}^{n}+\mathbf{S}^{n+1}
$$

where the discrete source takes the form (3). The term $\Delta \mathrm{t}$ has been added with a view to keep unities consistent. The source starts emitting at time $t_{p}$ and therefore at this instant of time, we have

$$
\mathbf{u}^{p}=\mathbf{S}^{p}=s_{p} \Delta t \mathbf{u}_{s} .
$$

At the next instant of time $t_{p+1}$, the vector $\mathbf{u}^{\mathrm{p}+1}$ will be updated according to

$$
\mathbf{u}^{p+1}=\mathbf{P} \mathbf{u}^{p}+\mathbf{S}^{p+1}=s_{p} \Delta t \mathbf{P} \mathbf{u}_{s}+s_{p+1} \Delta t \mathbf{u}_{s}
$$

Proceeding like this until time $t_{p+k}=(p+k) \Delta t$ this field will generate in the domain $\Omega$, voltages and currents that can be computed by the formula

$$
\mathbf{u}^{p+k}=s_{p} \Delta t \mathbf{P}^{k} \mathbf{u}_{s}+s_{p+1} \Delta t \mathbf{P}^{k-1} \mathbf{u}_{s}+\cdots+s_{p+k} \Delta t \mathbf{u}_{s} .
$$

Let $a$ be the index corresponding to the location $\mathbf{z}_{\mathrm{a}}$ of the voltage source for the vector $\mathbf{u}$ containing all the discrete values of the voltages and currents in the network. Writing equation (4) at point $\mathbf{z}=\mathbf{z}_{\mathrm{a}}$, the unknown coefficients $\left(s_{p}, s_{p+1}, \ldots, s_{p+k}\right)^{T}$ that should cancel both the voltage $b_{k}=v\left(\mathbf{z}_{a}, t_{k}\right)$ produced by the initial source and the voltage produced by the source (2) should satisfy the equation

$$
\begin{aligned}
& s_{p} \Delta t \mathbf{P}^{k} \mathbf{u}_{s}(a)+s_{p+1} \Delta t \mathbf{P}^{k-1} \mathbf{u}_{s}(a)+\cdots+s_{p+k} \Delta t \mathbf{u}_{s}(a) \\
& +b_{p+k}=0, \text { for } k \in\{q, \ldots, f\} .
\end{aligned}
$$

This equation can be written as a matrix-vector product

$$
\mathbf{A s}=-\mathbf{b},
$$

with $\mathbf{s}=\left(s_{p}, s_{p+1}, \ldots, s_{p+f}\right)^{T}$

and $\mathbf{b}=\left(b_{p+q}, b_{p+q+1}, \ldots, b_{p+f}\right)^{T}$.

In order to build the matrix $\mathbf{A}$, a pulse is sent at time $t=p \Delta t$ at point $\mathbf{z}=\mathbf{z}_{s}$. Then we observe the voltage created at point $\mathbf{z}=\mathbf{z}_{a}$ by this pulse between time $t=(p+q) \Delta t$ and time $t=(p+f) \Delta t$. Those values constitute the first column of the matrix $\mathbf{A}$.

In general, the $k^{\text {th }}$ column of matrix $\mathbf{A}$ contains the values of the voltage $v$ at point $\mathbf{z}=\mathbf{z}_{a}$ created by a pulse sent at time $t=(p+k-1) \Delta t$ at point $\mathbf{z}=\mathbf{z}_{s}$ for $t$ ranging from $t=(p+q) \Delta t$ to $t=(p+f) \Delta t$. On the other hand, the vector $\mathbf{b}$ contains the values of the voltage $v$ at point $\mathbf{z}=\mathbf{z}_{a}$ obtained by the initial source for $t$ ranging from $t=(p+q) \Delta t$ to $t=(p+f) \Delta t$.

The linear system is then solved in the least square sense since the matrix $\mathbf{A}$ is not square, in general. The problem also requires some regularization. For the numerical examples of the next section, we use Tikhonov's regularization [4]. Other regularization techniques, based on the singular value decomposition for example, could equally be used. However we found that Tikhonov's regularization was efficient enough for our problem. 


\section{NUMERICAL VALIDATION}

As a first experiment, we apply the LCCF method described in the previous section to the unshielded coaxial transmission line network shown on Figure 2.

The left end of line 1 is matched and all the other load impedances of the network are open loads. Threedimensional coupling between the branches of the network by radiation are neglected. For all the numerical simulations, we use a home-made Finite-Difference Time Domain (FDTD) code to solve equations (1) for the voltages and the currents. However, since the proposed method is non-intrusive, any commercial software such as CST or HFSS could equally be used.

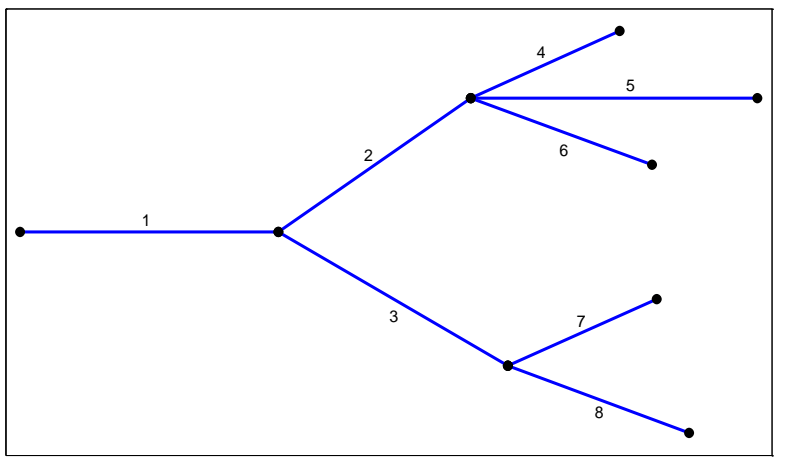

Fig. 2 : Network topology used for the numerical simulations.

The transmission lines are assumed to be lossless and they are characterized by distributed inductances $L=1 \times 10^{-6} \mathrm{H} / \mathrm{m}$ for all branches of the network. On the other hand, the distributed capacitance defined by $C_{i}=L / Z_{c, i}^{2}$, where $Z_{c, i}$ is the characteristic impedance of the line $i$, is line-dependant. The values, which were arbitrary chosen, are reported in Table 1.

Table 1. Characteristics of the transmission lines

\begin{tabular}{|c|c|c|}
\hline $\begin{array}{c}\text { Line } \\
\text { number }\end{array}$ & Length $(\mathrm{m})$ & $Z_{c, i}(\Omega)$ \\
\hline 1 & 0.09 & 50 \\
\hline 2 & 0.09 & 50 \\
\hline 3 & 0.10 & 30 \\
\hline 4 & 0.06 & 50 \\
\hline 5 & 0.10 & 50 \\
\hline 6 & 0.07 & 50 \\
\hline 7 & 0.06 & 50 \\
\hline 8 & 0.07 & 50 \\
\hline
\end{tabular}

As a first step, a voltage source is placed at a point $\mathbf{z}_{\mathrm{s}}$ located at $0.006 m$ from the left extremity of line 1 . This source emits a Gaussian signal of the form

$$
v\left(t, \mathbf{z}_{s}\right)=\exp \left(-\left[\frac{t-t_{0}}{\sigma}\right]^{2}\right),
$$

with $t_{0}=3 \times 10^{-10} s$ and $\sigma=8 \times 10^{-11}$. This source will generate a voltage at a point $\mathbf{z}_{a}$ located $0.07 \mathrm{~m}$ away from the left extremity of line 2, as shown on Figure 3.

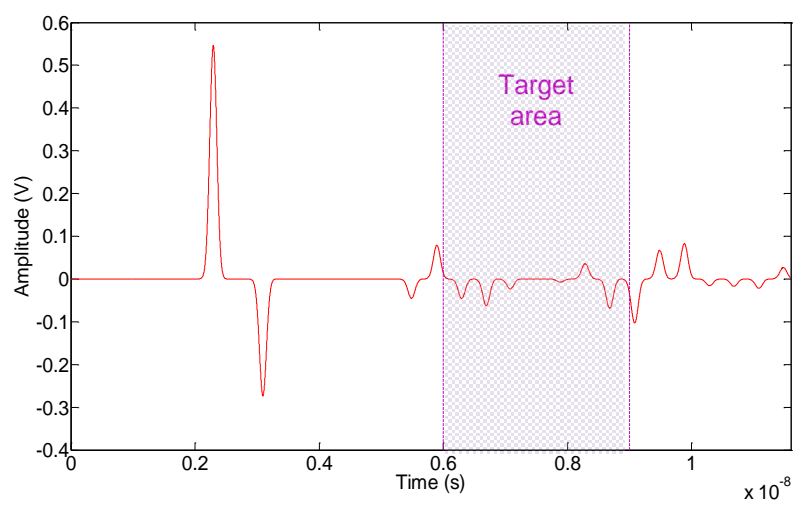

Fig. 3 : Voltage at point $\mathbf{z}_{a}$

For this simulation, the space and time discretization are $\Delta z=1 \times 10^{-4} \mathrm{~m}$ and $\Delta t=1 \times 10^{-12} \mathrm{~s}$, respectively. Those values are chosen to handle the maximum frequency in the network together with the CFL criterion that links the time step, the space discretization and the maximum wave speed in the network. Then, applying the LCCF method, we compute the form of the source that emits a signal at point $\mathbf{z}_{\mathrm{s}}$ to cancel the voltage at point $\mathbf{z}_{\mathrm{a}}$ for the period of time between $t_{p+q}=6000 \Delta t=6 \times 10^{-9} \mathrm{~s}$ and $t_{p+f}=9000 \Delta t=9 \times 10^{-9} \mathrm{~s}$. For that, the source will start emitting at time $t_{p}=3000 \Delta t=3 \times 10^{-9} \mathrm{~s}$. Figure 4 shows the profile of the voltage source computed with the LCCF method. We note that this signal has a complex shape and could not be guessed from the start.

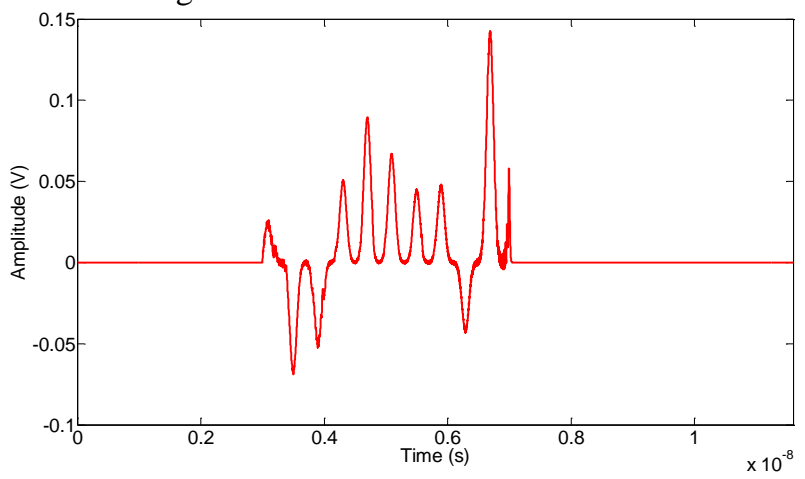

Fig. 4 : Voltage source computed with the LCCF method to cancel the voltage at point $\mathbf{z}_{a}$.

Then, in a second simulation, this signal is injected into the network after the initial Gaussian signal (equation (7)). The voltage obtained at point $\mathbf{z}_{a}$ for this new simulation is shown on Figure 5. We can clearly see the efficiency of the LCCF method since the voltage is zero on the interval $\left[t_{p+q}, t_{p+f}\right]$. Indeed, the average value of the voltage at point $\mathbf{z}_{\mathrm{a}}$ on that interval is $1.175 \times 10^{-9} \mathrm{~V}$. 


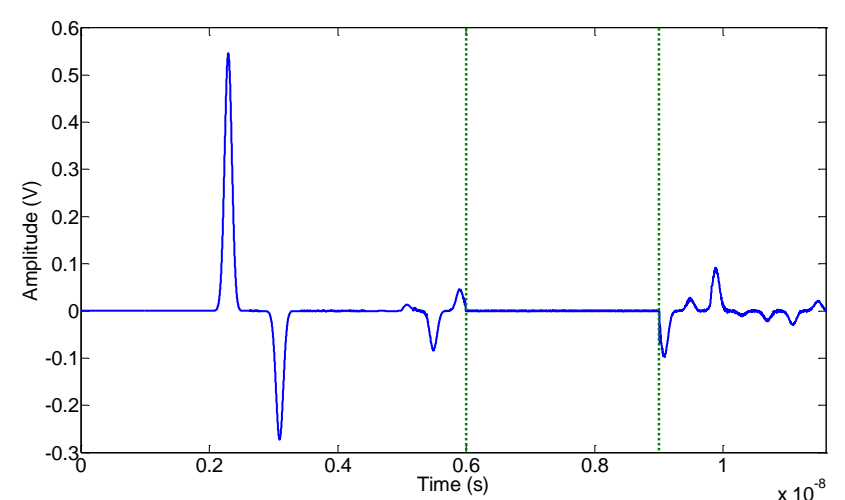

Fig. 5 : Voltage observed at point $\mathbf{z}_{a}$ by the source voltage shown on Figure 4.

Not surprisingly, the voltage source (Figure 4) has to start emitting before the voltage is canceled at point $\mathbf{z}_{\mathrm{a}}$. It also affects the voltage after time $t_{p+f}=9000 \Delta t=9 \times 10^{-9} \mathrm{~s}$ (compare Figure 3 with Figure 5).

As a second numerical experiment, we introduce a local modification of the characteristic of one of the lines of the network. For example, we set $L=1.1 \times 10^{-6} H$ for the RLCG cell located $0.06 m$ away from the left extremity of the line number 3 (this represents a $10 \%$ variation of the initial value of the inductance). The change of impedance may model a fault in the network at that point, and the objective is to be able to detect this fault. To achieve this, the signal (7) to be generated by the source shown on Figure 4 is sent. The resulting voltage at point $\mathbf{z}_{\mathrm{a}}$ is shown on Figure 6 for both the network without fault and the network with a fault on the inductance, for $t \in\left[t_{p+q}, t_{p+f}\right]$.

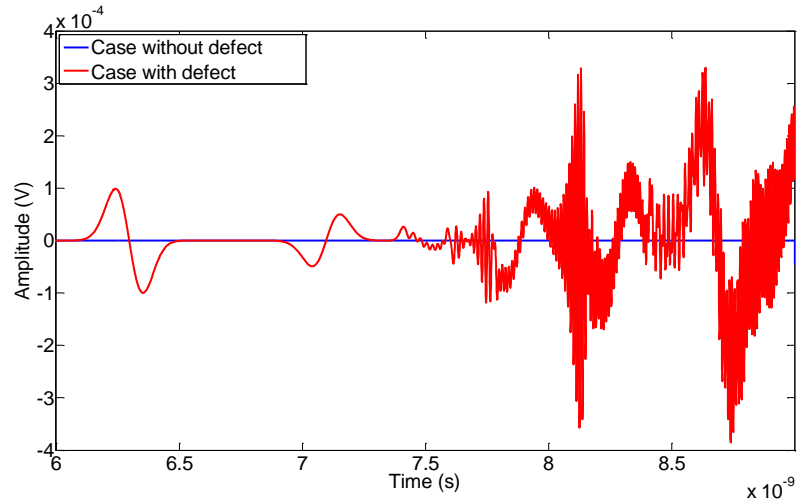

Fig. 6: Voltage observed at point $\mathbf{z}_{a}$ with and without defect.

We can see a clear demarcation between the two curves. In order to quantify this difference, we can compute the average amplitude of the voltage on this interval and we find $4.843 \times 10^{-5} V$ whereas it was $1.175 \times 10^{-9} V$ without fault in the network. Therefore, a slight $10 \%$ modification of the inductance in one cell of length $1 \times 10^{-4} \mathrm{~m}$ in the network, induces a considerable relative variation of the voltage at point $\mathbf{z}_{\mathrm{a}}$, for the particular source shown on
Figure 4. Thus, the LCCF can also be efficiently used as fault detection, since it can produce sources that are very sensitive to small changes in the network. In the next section, we will see how the same ideas can be adapted in an experimental framework. However, before that, it is important to test the robustness of the LCCF method with respect to noise measurement. This test is performed numerically by adding noise to the columns of the matrix A appearing in equation (6). This noise can be modeled by a random variable added to each element of the matrix A. The random variables are assumed to follow a Gaussian law with zero mean and a standard deviation equal to $5 \%$ of the deterministic values appearing in the matrix A

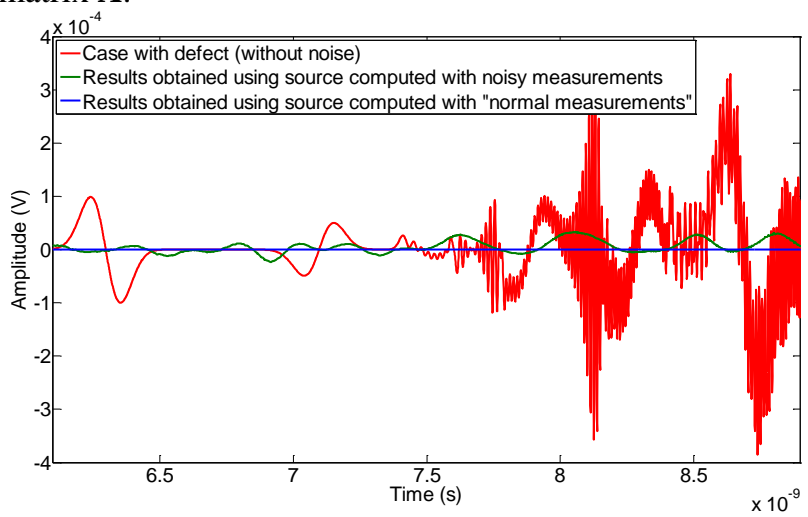

Fig. 7: Voltage observed at point $\mathbf{z}_{a}$ using a source computed from noisy measurements and non-noisy measurements.

The source voltage computed with this new sample matrix is then re-injected into the network and the resulting voltage at point $\mathbf{z}_{\mathrm{a}}$ is shown on Figure 7. Although the noise affects the voltage, its effect is still one order of magnitude below the effect of a fault in the network. Therefore, it should be possible to apply the LCCF method experimentally and this is what we do in the next section.

\section{EXPERIMENTAL RESULTS}

In order to experimentally validate the LCCF, we consider a network designed with coaxial cables. One end of the network is connected to an arbitrary waveform generator (AWG, Tektronix AFG 3021B) and another end is connected to a numerical oscilloscope (LeCroy WaveRunner 640Zi).

The experimental set up is shown on Figure 8 and Figure 9 gives a schematic representation of the network to be study. 


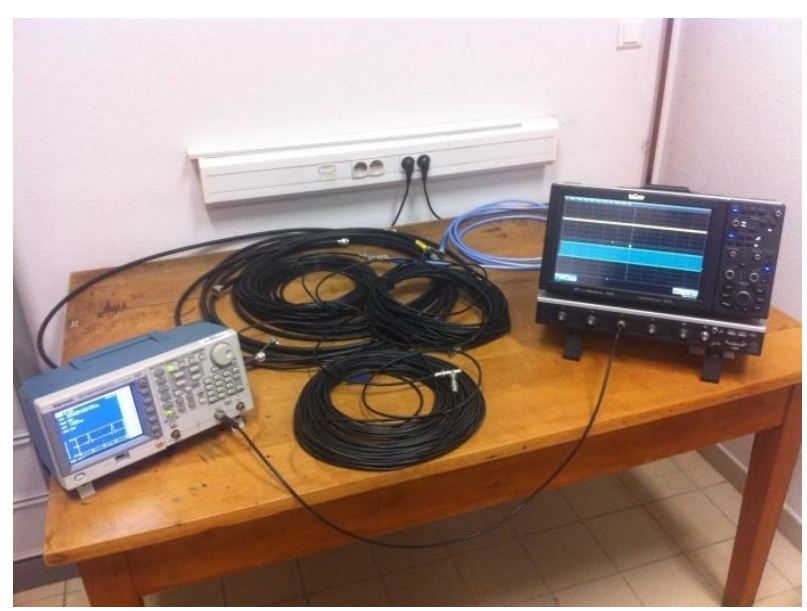

Fig. 8: Picture of the experimental set up. From left to right: the arbitrary waveform generator, the coaxial cable and the numerical oscilloscope.

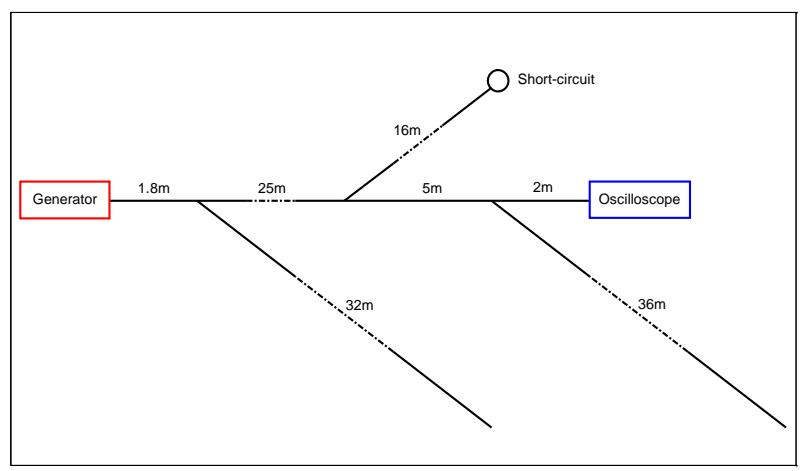

Fig. 9: Details of the network configuration for the experimental test.

Here, the purpose is to determine the profile of the voltage source that the AWG should generate in order to produce a specified voltage recorded by an oscilloscope at one end of the network.

The procedure to apply the LCCF method remains the same as previously seen for the numerical simulations. First, the AWG generates a pulse in the network and the oscilloscope records the resulting voltage. This given voltage is used to build the matrix A. As objective, we choose a voltage with a Gaussian shape. Then, the requested source can be obtained by solving equation (6). Note that the only difference with the purely numerical procedure described in the previous sections resides in the construction of the matrix $\mathbf{A}$, which is done from experimental data. However, only objectives of simple shapes are possible due to the very basic experimental hardware used here. Once computed, the voltage source is generated by the AWG and the resulting voltage at the other end of the network is recorded by the numerical oscilloscope. Figure 10 shows the recorded voltage (blue line) together with the objective voltage (red line).

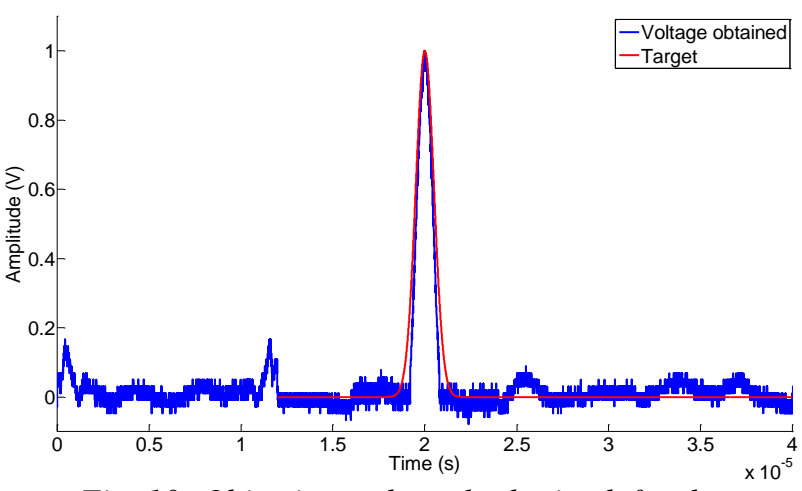

Fig. 10: Objective and result obtained for the experimental setting.

It clearly appears that the result matches very well with the objective over some period of time. With top-notch hardware, it is possible to generate higher frequency sources and obtaining more complex signals should be possible. However, one should bear in mind that only physically acceptable voltages can be obtained (i.e. they should satisfy equation (1)). Also, the number of possible reflections within the network grows with its size and one should expect to require a more complex source to produce the objective. On the other hand, it would be highly unrealistic to have a complex voltage that is different from the source for a simple matched line.

We now introduce a modification in the transmission line network, which is assumed to model a fault, and we now test if the source computed by the LCCF method is able to produce a voltage that is significantly different from the objective. For that, we remove the short circuit located at the end of the $16 \mathrm{~m}$ branch. Then, we inject the source previously computed in the modified network and we observe on Figure 11 that the voltage does not match the objective.

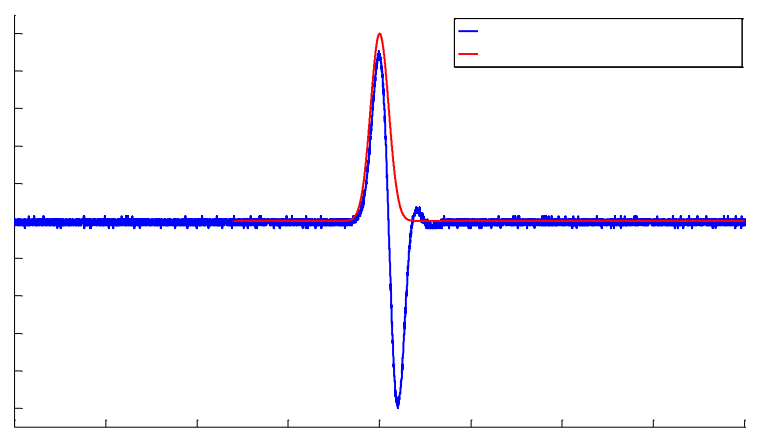

Fig. 11: Response of the network with and without defect.

For this example, the modification of the transmission line network was major, and any observable output would be significantly affected, even with sources not computed with the LCCF method. In a view of using more sophisticated AWG that can deal with higher frequencies, the experimental application of the LCCF method should 
enable the detection of soft faults with a high sensitivity, as shown by the numerical experiments.

\section{CONCLUSION}

In this article, an extension of the LCCF method to transmission lines problems was carried out. We showed that it is possible to construct a voltage source, which, injected at one point of the network, will produce a specified voltage in another point of the same network. Then, by observing that the obtained voltage is highly sensitive to the physical properties of the network, this technique can also be efficiently used to detect faults within a network. This idea was successfully tested experimentally. The LCCF method presented in this paper can easily be adapted to treat more complex problems: imposing a time domain voltage on more than one point or treating networks with highly complex topology, for example.

Other EMC applications to this general method may be considered in the future. Amongst them, we can think of faults localization in a network or adapting voltages/currents in a network in order to satisfy some threshold limit.

\section{REFERENCES}

[1] J. Benoit, C. Chauvière, P. Bonnet, "Une Alternative au Retournement Temporel pour Focaliser Spatialement et Temporellement une Onde Electromagnétique", $16^{\mathrm{eme}}$ Colloque International sur la Compatibilité ElectroMagnétique (CEM 2012), Rouen, 2012.

[2] J. Benoit, C. Chauvière et P. Bonnet, "Source Identification in time domain electromagnetics", Journal of Computational Physics Vol. 231, Issue 8, 2012, pp 3446-3456.

[3] C.R. Paul, "Analysis of Multiconductor Transmission Lines", Wiley-Interscience, ISBN 9780470131541, 2008.

[4] A. N. Tychonoff, "Solution of incorrectly formulated problems and the regularization method", Soviet Mathematics 4, pp. 1035-1038, 1963.

[5] T.K. Sarkar, A. Taaghol, "Near-field to near/farfield transformation for arbitrary near-field geometry, utilizing an equivalent electric current and MoM", IEEE Trans. Antennas Propag. 47 (3) (1999) 566-573.

[6] J.E. Hansen (Ed.), "Spherical Near-Field Antenna Measurements, IEE Electromagnetic Waves Series", vol. 26, Peter Peregrinus Ltd., Stevenage, UK, 1988, ISBN 0-86341-110-X.

[7] F. de Daran, J. Chollet-Ricard, F. Lafon, and O. Maurice, "Prediction of the field radiated at one meter from $P C B$ 's and microprocessors from near EM field cartography," Proc. IEEE Int. Symp.
Electromagn. Compat., Vol. 1, 479-482, Istanbul, Turkey, May 2003.

[8] P. Petre and T. K. Sarkar, "Planar near-field to farfield transformation using an equivalent magnetic current approach," IEEE Trans. Antennas Propag., Vol. 40, No. 11, 1348-1355, Nov. 1992.

[9] K.S. Yee, "Numerical solution of initial boundary value problems involving Maxwell's equations in isotropic media". IEEE Transactions on Antennas and Propagation 14: 302-307, 1966.

[10] M. Fink, "Time reversal of ultrasonic Fields: part I. Basic principles", IEEE Trans. Ultrason. Ferro-elect. Freq. Control, Vol. 39, No. 5, pp. 555-566, Sept. 1992.

[11] G. Lerosey, J. de Rosny, A. Tourin, A. Derode, G. Montaldo, and M. Fink, "Time Reversal of Electromagnetic Waves", Physical Review Letters 92-19, May 2004.

[12] P. Bonnet, X. Ferrieres, B. L. Michielsen, P. Klotz, J. L. Roumiguieres, "Finite-volume time domain method", In Time Domain Electromagnetics, S. M. Rao (ed.). Academic Press: San Diego, CA, 1999.

[13] J. S. Hesthaven, T. Warburton, "Nodal high-order methods on unstructured grids: I. Time-domain solution of Maxwell's equations" Journal of Computational Physics Vol. 181, Issue 1, 2002, pp 186-221.

[14] A. Ungureanu, Yan Fu, Tan-Phu Vuong, F. Ndagijimana, "Electromagnetic source synthesis by reversed-TLM method" IEEE International Microwave Symposium, Baltimore, 2011.

[15] M.K. Smail, T. Hacib, L. Pichon, F. Loete, "Detection and location of defects in wiring networks using time domain reflectometry and neural networks", IEEE Transactions on Magnetics, Vol. 47 No.5, 2011, pp 1502-1505.

[16] L. Abboud, A. Cozza, L. Pichon, "A matched-pulse approach for soft-fault detection in complex wire networks", IEEE Transactions on Instrumentation and Measurement, Vol. 61, no. 6, pp 1719-1732, 2012.

[17] M. Franchet, N. Ravot, O. Picon, "Soft Fault Detection in Cables using the Cluster TimeFrequency Domain Reflectometry", IEEE Electromagnetic Compatibility Magazine - Volume 2, pp 54-69, 2013.

[18] C. Obiekezie, D. W. P Thomas, A. Nothofer, S. Greedy, L. R. Arnaut and P. Sewell, "A Technique for Analyzing EMC Shielding Effectiveness Based on Near-Field Measurement and Equivalent Dipole Modeling," in Proc. 2013 International Symposium on Electromagnetic Compatibility pp. 699-704.

[19] Y. Liu, B. Ravelo and P. Fernandez-Lopez, "Modeling of Magnetic Near-Field Radiated by Electronic Devices Disturbed by Complex Transient 
Signals", Applied Physics Research (APR), Vol. 4, No. 1, 2012, pp. 3-18.

[20] A. K. Jastrzebski, Y. Liu and B. Ravelo, "Characterisation and Modelling of Near-Field Radiated Emissions in the Time-Domain”, APEMC 2012, pp. 201-204.

[21] L. El Sahmarany, L. Berry, K. Kerroum, F. Auzanneau and P. Bonnet, "Time reversal forwiring diagnosis", Electronics letters, September 2012

[22] L. El Sahmarany, L. Berry, N. Ravot, F. Auzanneau and P. Bonnet, "Time reversal for soft faults diagnosis in wire networks", Progress In Electromagnetics Research (PIER), May 2013 